\title{
Hidden SUSY from precision gauge unification
}

\author{
Sven Krippendorf, ${ }^{1, *}$ Hans Peter Nilles, ${ }^{1, \dagger}$ Michael Ratz, ${ }^{2, \ddagger}$ and Martin Wolfgang Winkler ${ }^{3,8}$ \\ ${ }^{1}$ Bethe Center for Theoretical Physics and Physikalisches Institut der Universität Bonn, Nussallee 12, 53115 Bonn, Germany \\ ${ }^{2}$ Physik-Department T30, Technische Universität München, James-Franck-Straße, 85748 Garching, Germany \\ ${ }^{3}$ Deutsches Elektronen-Synchroton DESY, Notkestrasse 85, 22607 Hamburg, Germany
}

(Received 10 June 2013; published 26 August 2013)

\begin{abstract}
We revisit the implications of naturalness and gauge unification in the minimal supersymmetric standard model. We find that precision unification of the couplings in connection with a small $\mu$ parameter requires a highly compressed gaugino pattern as it is realized in mirage mediation. Due to the small mass difference between the gluino and lightest supersymmetric particle (LSP), collider limits on the gluino mass are drastically relaxed. Without further assumptions, the relic density of the LSP is very close to the observed dark matter density due to coannihilation effects.
\end{abstract}

DOI: 10.1103/PhysRevD.88.035022

PACS numbers: $12.60 . \mathrm{Jv}$

\section{INTRODUCTION}

Two key motivations for studying low-energy supersymmetry are the possibility for a natural solution to the electroweak hierarchy problem [1] and gauge coupling unification [2]. Given this input of a natural solution to the electroweak hierarchy problem and gauge coupling unification, we analyze in this article their effect on the spectrum of supersymmetric soft masses. Here we concentrate on the minimal supersymmetric extension of the standard model (MSSM).

If one ponders the question whether or not gauge couplings really unify in the MSSM, it turns out that there is a non-negligible dependence on the pattern of supersymmetry breaking. In particular, in some of the most popular scenarios, such as the constrained MSSM (CMSSM), gauge coupling unification is not precise given soft masses around the weak scale. This refers to the fact that the strong coupling $\alpha_{3}=g_{3}^{2} /(4 \pi)$ turns out to be about 3\% smaller than $\alpha_{1}$ and $\alpha_{2}$ at $M_{\mathrm{GUT}}$, which is defined as the scale where $\alpha_{1}$ and $\alpha_{2}$ unify.

Of course, this discrepancy of the couplings at $M_{\mathrm{GUT}}$ may originate from some thresholds at the high scale [3] (see also S. Raby in [4]). However, here we would like to discuss precision gauge coupling unification (PGU) in the absence of high-scale threshold corrections ${ }^{1}$ and determine the consequences for the spectrum of soft masses.

Several examples of spectra achieving PGU have been discussed in the literature [5-8]. Most of these are now excluded by the nonobservation of superpartners at the LHC or by the relatively large mass of the Higgs boson. The consideration of naturalness leads us to the choice of a

\footnotetext{
*krippendorf@th.physik.uni-bonn.de

†nilles@th.physik.uni-bonn.de

†michael.ratz@tum.de

§martin.winkler@desy.de

${ }^{1}$ The size of these threshold corrections is ultimately set by the UV theory of choice. For a bottom-up analysis we take it as a UV parameter which we here assume to be vanishing.
}

small $\mu$ parameter which (further) narrows down viable soft mass spectra. Remarkably, we are led to a highly compressed pattern of gaugino and Higgsino masses at or below the $\mathrm{TeV}$ scale, while the scalar superpartners can be out of reach for the LHC experiment. As the LHC sensitivity is drastically reduced in case of a small mass splitting between the gluino and the lightest supersymmetric particle (LSP), PGU provides an attractive explanation of why SUSY has not been discovered so far.

The required spectrum of gaugino masses can be accommodated in mirage mediation and as such is directly connected to UV scenarios arising within string theory [9-13]. Fixing the ratio of gaugino masses with PGU opens interesting windows towards explicit UV realizations of mirage mediation.

Further, the problem of dark matter overproductionusually arising in SUSY models with a bino LSP - can naturally be solved by a compressed gaugino spectrum. We find that in mirage mediation, PGU is intimately linked to the occurrence of coannihilations. These allow for a consistent explanation of dark matter in terms of the lightest neutralino.

This article is organized as follows: in section II we perform a model-independent analytical discussion of gauge coupling unification. In section III, we study whether or not PGU can be achieved in realistic SUSY models taking the CMSSM and mirage mediation as examples. Then, we turn to the LHC and dark matter phenomenology of models with successful PGU in section IV, before concluding in section V.

\section{THE EFFECTIVE SUSY THRESHOLD SCALE}

The MSSM gauge couplings evaluated at the scale of grand unification can be written as [14]

$\frac{1}{g_{i}^{2}\left(M_{\mathrm{GUT}}\right)}=\frac{1}{g_{i}^{2}\left(M_{Z}\right)}-\frac{b_{i}^{\mathrm{MSSM}}}{8 \pi^{2}} \ln \left(\frac{M_{\mathrm{GUT}}}{M_{Z}}\right)+\frac{1}{g_{i, \mathrm{Thr}}^{2}}+\Delta_{i}$ 
where the $b_{i}^{\text {MSSM }}$ denote the standard MSSM beta function coefficients, $\left(b_{1}, b_{2}, b_{3}\right)=(33 / 5,1,-3)$, while $\Delta_{i}$ includes two-loop effects, threshold corrections related to the heavy standard model fields as well as a possible term required for the transition between renormalization schemes - the gauge couplings $g_{i}^{2}\left(M_{Z}\right)$ are typically given in the $\overline{\mathrm{MS}}$ rather than the $\overline{\mathrm{DR}}$ scheme. The threshold corrections within the MSSM read [14]

$$
\frac{1}{g_{i, \mathrm{Thr}}^{2}}=\sum_{\eta} \frac{b_{i}^{\eta}}{8 \pi^{2}} \ln \left(\frac{M_{\eta}}{M_{Z}}\right)
$$

where the sum runs over all sparticles and heavy Higgs fields with mass $M_{\eta}$ evaluated at the low scale and $b_{i}^{\eta}$ denoting the contribution of the particle $\eta$ to the $i$ th $\beta$-function coefficient (cf. [15] for the exact expressions of $b_{i}^{\eta}$ in the MSSM). ${ }^{2}$

To arrive at a consistent picture of unification, the $g_{i}$ are required to meet in a single point. Assuming the absence of threshold corrections induced by heavy fields of a grand unified theory (GUT), this implies that the masses of the MSSM fields must arrange such that their overall threshold to the $g_{i}$ allows for PGU.

To get a first impression on the effect of soft masses on gauge coupling unification, let us make the ad hoc assumption that all sparticles as well as the heavy Higgs doublet have a common mass $T_{\text {SUSY }}$ which we call the SUSY threshold scale. In this case, the overall threshold correction (2) to the gauge couplings takes a very simple form. We find

$$
\frac{1}{g_{i, \mathrm{Thr}}^{2}}=\frac{b_{i}^{\mathrm{MSSM}}-b_{i}^{\mathrm{SM}}}{8 \pi^{2}} \ln \left(\frac{T_{\mathrm{SUSY}}}{M_{Z}}\right),
$$

where $b_{i}^{\mathrm{SM}}$ stands for the beta function coefficients of the standard model. Now we can determine the SUSY threshold scale for which the gauge couplings meet exactly. To quantify the deviation from PGU, we follow $[7,16]$ and introduce

$$
\epsilon_{3}=\frac{g_{3}^{2}\left(M_{\mathrm{GUT}}\right)-g_{1,2}^{2}\left(M_{\mathrm{GUT}}\right)}{g_{1,2}^{2}\left(M_{\mathrm{GUT}}\right)},
$$

where we define $M_{\mathrm{GUT}}$ as the scale at which $g_{1}$ and $g_{2}$ meet.

In Fig. 1 we depict $\epsilon_{3}$ as a function of the SUSY threshold scale. For the determination of $\epsilon_{3}$, we have used Softsusy (version 3.3.2) [17], where we set the strong coupling strength to $\alpha_{s}\left(M_{Z}\right)=0.1184 \pm 0.0007$ [18] and $\tan \beta=10$. It can be seen that the three gauge couplings would exactly meet in a single point if all superpartners as well as the heavy Higgs doublet had a common mass $T_{\text {SUSY }} \simeq 2 \mathrm{TeV}$. Note that the required value of $T_{\mathrm{SUSY}}$ has a very mild dependence on $\tan \beta$ which only affects the gauge coupling at the two-loop level.

\footnotetext{
${ }^{2}$ Here we neglect two-loop contributions to the thresholds.
}

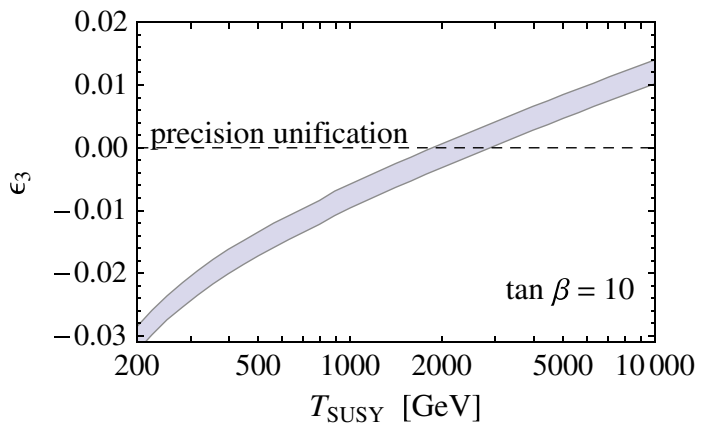

FIG. 1 (color online). Deviation from PGU as a function of $T_{\text {SUSY }}$. The width of the band corresponds to the $1 \sigma$ experimental error in $\alpha_{s}\left(M_{Z}\right)$.

Turning to realistic SUSY models, despite the fact that not all superpartners have a common mass, it is still possible to define an effective SUSY threshold scale $T_{\text {SUSY }}$ through the relation

$$
\begin{aligned}
& \frac{b_{i}^{\mathrm{MSSM}}-b_{i}^{\mathrm{SM}}}{8 \pi^{2}} \ln \left(\frac{T_{\mathrm{SUSY}}}{M_{Z}}\right) \\
& \equiv \sum_{\eta} \frac{b_{i}^{\eta}}{8 \pi^{2}} \ln \left(\frac{M_{\eta}}{M_{Z}}\right)+\left(c_{1}+c_{2} b_{i}^{\mathrm{MSSM}}\right)
\end{aligned}
$$

with the same ( $\left.T_{\mathrm{SuSY}}, c_{1}, c_{2}\right)$ for all three standard model gauge groups. Note that the term $c_{1}$ accounts for an overall shift of all $g_{i}\left(M_{\mathrm{GUT}}\right)$, while the $c_{2}$ term results in a change of $M_{\mathrm{GUT}}$.

Therefore $T_{\text {SUSY }}$ has a very simple interpretation: a SUSY spectrum with an effective SUSY threshold scale $T_{\text {SUSY }}$ has the same effect on gauge coupling unification as a degenerate spectrum with all superpartners having this common mass, up to a change of $M_{\mathrm{GUT}}$ and/or the unified gauge coupling.

The effective SUSY threshold scale takes the form ${ }^{3}$

$$
T_{\text {SUSY }}=\frac{m_{\tilde{W}}^{32 / 19} m_{\tilde{h}}^{12 / 19} m_{H}^{3 / 19}}{m_{\tilde{g}}^{28 / 19}} X_{\text {sfermion }},
$$

where $m_{\tilde{W}}, m_{\tilde{h}}, m_{H}$ and $m_{\tilde{g}}$ denote the mass of the wino, the Higgsino, the heavy MSSM Higgs and the gluino, respectively. The sfermion contribution reads

$$
X_{\text {sfermion }}=\prod_{i=1 \ldots 3}\left(\frac{m_{\tilde{L}^{(i)}}^{3 / 19}}{m_{\tilde{D}^{(i)}}^{3 / 19}}\right)\left(\frac{m_{\tilde{Q}_{L}^{(i)}}^{7 / 19}}{m_{\tilde{E}^{(i)}}^{2 / 19} m_{\tilde{U}^{(i)}}^{5 / 19}}\right),
$$

where the masses of the sfermions appear in a selfexplanatory notation. As we will show explicitly in the next section, $X_{\text {sfermion }} \simeq 1$ whenever the sfermion masses are universal within a $\mathrm{SU}(5)$ multiplet at the high scale which is expected to hold in a GUT model. The mass splitting of sleptons and squarks through renormalization

\footnotetext{
${ }^{3} \mathrm{~A}$ simplified version of this formula was discussed in [14].
} 
group running does not affect this conclusion. Hence, the sfermions leave no direct effect on PGU.

Note that the insensitivity of gauge coupling unification to the scale of sfermion masses is due to the completeness of these GUT multiplets. Only split GUT multiplets give nontrivial contributions to $T_{\text {SUSY }}$ [cf. equation (6)]. This is also utilized in models of split supersymmetry [19]. It is also interesting that the decoupling of complete generations of soft scalar masses, e.g. the first and second generation, does not affect PGU, allowing for a realization of "natural supersymmetry" with a light third generation of soft scalar masses.

\section{GAUGE COUPLING UNIFICATION IN MSSM MODELS}

As can be seen from equation (6), $T_{\text {SusY }}$ mainly depends on the Higgsino mass and on the mass ratio $m_{\tilde{W}}: m_{\tilde{g}}$. In the following, after discussing the case of uncompressed gaugino masses, we shall show how a change in this mass ratio can lead to PGU.

\section{A. Uncompressed gaugino mass spectra}

In models with gravity mediated SUSY breaking, the gaugino masses are typically expected to unify at the GUT scale $M_{\mathrm{GUT}}$. Taking $m_{1 / 2}$ to be the universal gaugino mass at the GUT scale, the physical mass of wino and gluino can be approximated as

$$
m_{\tilde{W}} \simeq 0.9 m_{1 / 2} \text { and } \quad m_{\tilde{g}} \simeq 2.5 m_{1 / 2} .
$$

In turn the effective SUSY threshold scale reads

$$
T_{\text {SUSY }} \simeq 0.3\left(m_{\tilde{h}}^{12} m_{1 / 2}^{4} m_{H}^{3}\right)^{1 / 19} X_{\text {sfermion }} .
$$

In the limit where the sfermions are substantially heavier than the gauginos, sfermion masses are only weakly affected by renormalization group equation (RGE) running. As long as the sfermion masses are universal within SU(5) multiplets at the high scale, we find $X_{\text {sfermion }} \simeq 1$. Even if gaugino masses are non-negligible and the masses of squarks and sleptons are split by RGE running, their effect on gauge coupling unification remains small. This is illustrated in Fig. 2 where we depict $X_{\text {sfermion }}$ in the CMSSM for a fixed gaugino mass $m_{1 / 2}$ and a varying scalar mass $m_{0}$. It can be seen that $X_{\text {sfermion }}$ never deviates by more than a few percent from 1 .

Taking $X_{\text {sfermion }}=1$, we can determine the Higgsino mass which is required to obtain PGU, i.e. $T_{\mathrm{SUSY}} \simeq$ $2 \mathrm{TeV}$. We find

$$
m_{\tilde{h}} \simeq 20 \mathrm{TeV} \times\left(\frac{\mathrm{TeV}}{m_{1 / 2}}\right)^{1 / 3}\left(\frac{\mathrm{TeV}}{m_{H}}\right)^{1 / 4} .
$$

Due to the small exponents of the last two terms, Higgsino masses as large as $m_{\tilde{h}}=\mathcal{O}(10 \mathrm{TeV})$ are required for gauge coupling unification, even if we take $m_{1 / 2}$ and $m_{H}$ in the multi-TeV range. As is well known, such heavy Higgsinos

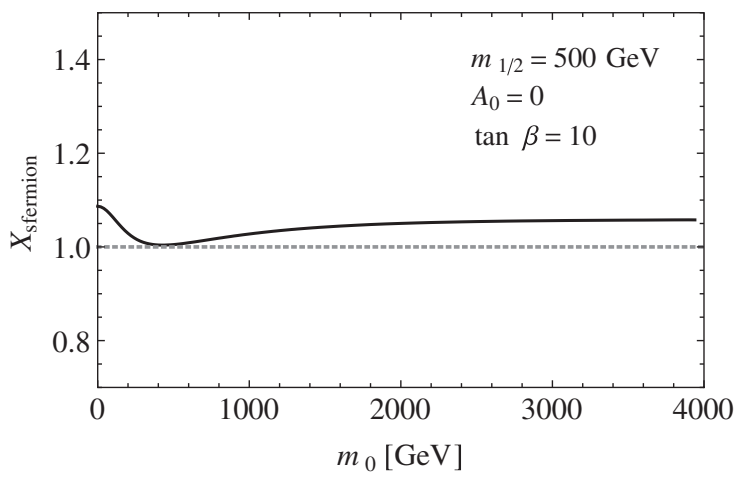

FIG. 2. Effect of sfermions on gauge coupling unification in the CMSSM for varying $m_{0}$.

lead to "unnaturally" large fine-tuning as can be seen from the mass of the $Z$ boson which-at tree level-can be written as $M_{Z} \simeq-2 m_{h_{u}}^{2}-2|\mu|^{2}$ for $\tan \beta \gg 1$. In the case of a large Higgsino mass, severe cancellations between $m_{h_{u}}$ and $\mu$ are required.

We will therefore now turn to a class of models in which PGU can be accommodated more naturally.

\section{B. Compressed gaugino mass spectra}

The effective $T_{\text {SUSY }}$ can be enhanced if one increases the wino mass relative to the gluino mass. Specifically, we now discuss the possibility of realizing PGU by nonuniversal gaugino masses which can be accommodated in soft mass spectra arising from UV completions of the MSSM. To obtain nonuniversal gaugino masses, an obvious possibility is to use anomaly mediated contributions. While in pure anomaly mediation the hierarchy between the gluino and wino is even larger than in gravity mediation, more compressed gaugino spectra can be obtained in schemes with mixed gravity/ anomaly mediation, also referred to as mirage scheme.

At first sight, having gravity and anomaly mediation at similar strength appears as an ad hoc assumption. But note that this naturally occurs in a wide class of string models due to the interplay between moduli stabilization and SUSY breaking. Examples of such mixed mediation schemes have been discussed in the context of type IIB string theory with moduli stabilization following Kachru, Kallosh, Linde and Trivedi (KKLT) [9,10,12,20-22], in the context of heterotic string theory [13,23-25], and in the context of $G_{2}$ compactifications [26].

In mirage mediation, the gaugino masses at the high scale can be written as

$$
M_{i}=\frac{m_{3 / 2}}{16 \pi^{2}}\left(\varrho+b_{i}^{\mathrm{MSSM}} g^{2}\right)
$$

where $m_{3 / 2}$ denotes the gravitino mass and $\rho$ parametrizes the gravity mediated contribution to the gaugino masses; in the limit of vanishing $\varrho$ pure anomaly mediation is recovered. Notice that $\left|M_{3}\right|<\left|M_{2}\right|$ at the GUT scale unless for $\varrho \leqq 0.5$. The gaugino masses at the low scale can be approximated as 


$$
\begin{aligned}
m_{\tilde{B}} & \simeq 0.45(\varrho+3.3) \frac{m_{3 / 2}}{16 \pi^{2}}, \\
m_{\tilde{W}} & \simeq 0.9(\varrho+0.5) \frac{m_{3 / 2}}{16 \pi^{2}}, \\
m_{\tilde{g}} & \simeq 2.4(\varrho-1.5) \frac{m_{3 / 2}}{16 \pi^{2}} .
\end{aligned}
$$

The gaugino spectrum is thus typically more compressed than in models with gaugino mass unification. In the regime $0.8 \lesssim \varrho \lesssim 2.5$ the gluino is even the lightest gaugino.

In the context of mirage mediation, the scalar masses and trilinear terms are model dependent. The former are of the order of the gravitino mass unless in case of sequestering. The trilinear soft terms are typically suppressed compared to the gravitino mass and can thus be neglected. In this study we assume sfermion masses in the multi-TeV regime as suggested by the KKLT and heterotic models with $F$-term uplifting [12,13,27,28].

In order to avoid a naturalness problem, we should require that $m_{h_{u, d}}=m_{\tilde{Q}_{L}^{(3)}}=m_{\tilde{U}^{(3)}}$ approximately hold the GUT scale. ${ }^{4}$ In this case, the RGE trajectory of $m_{h_{u}}$ exhibits the well-known focus point [29,30] such that multi-TeV scalars are not unnatural. In the focus point scenario $m_{h_{u}}$ is driven to a very small value at the low scale, while $m_{h_{d}}$ is not considerably affected by RGE running. This implies $m_{H} \simeq m_{h_{d}}$ for the physical mass of the heavy MSSM Higgs as well as a suppressed $\mu$ term [29]. Therefore, the above assumption is equivalent to $m_{H}=m_{\tilde{Q}_{L}^{(3)}}=m_{\tilde{U}^{(3)}} \gg \mu$ up to subleading corrections. As the running of the gauge couplings depends on $\mu$ rather than the Higgs soft masses this form of the input is more suitable for our purposes. To be specific we set

$$
m_{H}=m_{i} \equiv m_{0}
$$

where $m_{i}$ denotes the mass of the sfermion $i$ at the high scale. We are thus left with the five free parameters $m_{3 / 2}, \varrho$, $m_{0}, \mu$ and $\tan \beta$. To avoid excessive fine-tuning we restrict our attention to $\mu \leq 2 \mathrm{TeV}$. Further, for any point in the parameter space, we eliminate $m_{0}$ by requiring that the mass of the light MSSM Higgs is $m_{h}=126 \mathrm{GeV}$, which typically leads to $m_{0} \sim 15 \mathrm{TeV}$. We should point out that our conclusions are insensitive to the assumptions in the scalar sector as the latter hardly affect gauge coupling unification. Thus our discussions are also valid in schemes where some of the complete sfermion families receive a mass $\sim m_{3 / 2}$ which typically is larger than $m_{0}$.

We now can ask what constraints on the parameter space arise from imposing PGU. In Fig. 3, we present the parameter region consistent with PGU for fixed $m_{3 / 2}$ and $\tan \beta$. Precision unification clearly favors a compressed

\footnotetext{
${ }^{4}$ This is a very reasonable assumption as e.g. in the heterotic models, these fields have the same localization properties (see discussion in $[23,24])$.
}

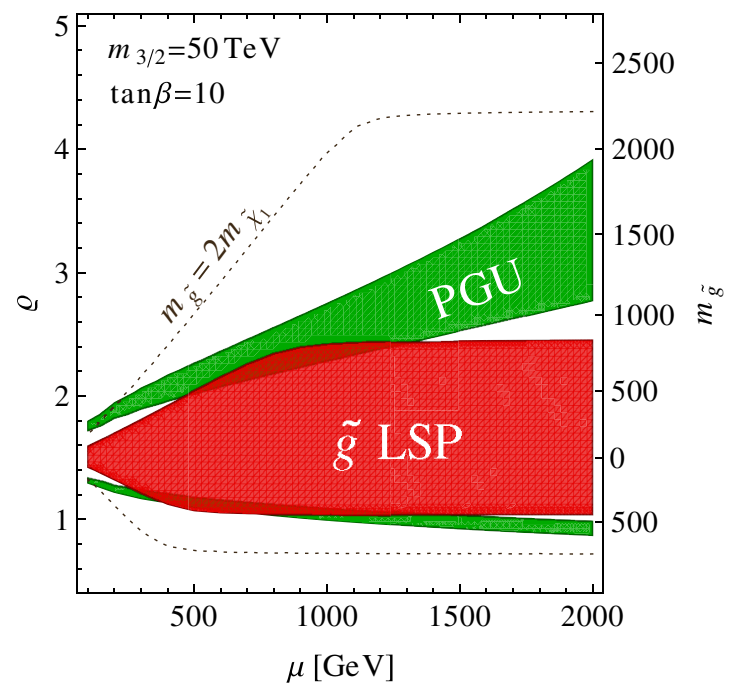

FIG. 3 (color online). Parameter scan in the $(\mu, \varrho)$ plane for fixed $m_{3 / 2}=50 \mathrm{TeV}$. The region where the gauge couplings unify precisely within the experimental error on the strong coupling are shown in green. The red region exhibits a gluino LSP. The dotted contour indicates where the mass ratio between gluino and LSP becomes 2.

spectrum, i.e. a spectrum where the mass difference between gluino and LSP is small. For $1.1<\varrho<2.4$, the gluino is the LSP, unless the Higgsino becomes even lighter (shrinking of the red band at the left side in Fig. 3). The two regions in parameter space consistent with PGU correspond to the two regions where the mass ratio of wino and gluino gives rise to the required effective SUSY threshold scale.

There is only a weak dependence on the overall scale of gaugino masses which is set by $m_{3 / 2}$. This is due to the fact that the SUSY threshold scale $T_{\text {SUSY }} \propto m_{\tilde{W}}^{32 / 19} / m_{\tilde{g}}^{28 / 19}$, i.e. the dependence on $m_{3 / 2}$ almost cancels out. Therefore, our results remain qualitatively unchanged for different choices of $m_{3 / 2}$. Similarly, PGU only shows a weak dependence on $\tan \beta$. For illustration, we have included scans with varying $m_{3 / 2}$ and $\tan \beta$ in the Appendix.

\section{IMPLICATIONS FOR THE LHC AND DARK MATTER}

\section{A. LHC discovery potential}

We can now turn to the implications of PGU for SUSY searches at the LHC. As described in the previous section, this naturally leads us to models with mirage mediation where PGU can naturally be accommodated with a small $\mu$.

Within the mirage scheme, solid predictions on the superpartner spectrum can be made. While the scalar superpartners are typically outside the reach of the LHC, the gauginos and Higgsinos remain relatively light. We cannot predict the hierarchy among gauginos and 
Higgsinos, but there is a general trend that PGU prefers a spectrum in which the mass difference between the gluino and the lightest neutralino is small.

Such compressed SUSY models are much more difficult to detect at the LHC than ordinary SUSY models like the CMSSM (see e.g. [31-33]). While there is still a strong gluino pair production, the subsequent decay of gluinos yields only a small amount of visible energy. In the experimental searches, a sizable fraction of the potential signal events is rejected due to too soft jets. In the case of extreme compression, only events with initial state radiation can pass the event cuts, and the signal acceptance is typically well below $1 \%$ [32].

If the gluino is the next-to-lightest supersymmetric particle, the relevant gluino decay modes read

$$
\begin{aligned}
& \tilde{g} \rightarrow q \bar{q}+\tilde{\chi}_{1}, \\
& \tilde{g} \rightarrow g+\tilde{\chi}_{1},
\end{aligned}
$$

where $\tilde{\chi}_{1}$ denotes the lightest neutralino. The first process proceeds via an off-shell squark, the second via a quark/ squark loop. Additional decay modes open up if further neutralinos or charginos are lighter than the gluino.

It is instructive to determine the regime of gluino and (lightest) neutralino masses preferred by PGU. For this purpose, we have generated a large data sample with random choice of input parameters in the intervals

$$
\begin{array}{llrl}
\varrho & =0.5-30, & m_{3 / 2} & =\frac{40-200 \mathrm{TeV}}{\varrho}, \\
\mu & =0.1-2 \mathrm{TeV}, & \tan \beta & =10-50 .
\end{array}
$$

Note that the considered range of $m_{3 / 2}$ corresponds to a gravity mediated contribution to the gaugino masses of $0.25-1.25 \mathrm{TeV}$.

In Fig. 4, we provide a scatter plot of those parameter sets which lead to successful PGU in the $\left(m_{\tilde{g}}, m_{\tilde{\chi}_{1}}\right)$ plane. To guide the eye, we have also included the present limits on the gluino mass from the ATLAS search for jets plus missing energy at $\int \mathrm{d} t L=5.8 \mathrm{fb}^{-1}$ [34] and from the CMS search for $b$-jets plus missing energy at $\int \mathrm{d} t L=$ $19.4 \mathrm{fb}^{-1}[35] .^{5}$ Both analyses are based on a simplified model with just the gluino and the LSP assuming 100\% branching of the process (14a). CMS additionally requires the final state quarks to be bottoms. Both limits are not strictly applicable as the considered decay modes do not necessarily dominate in the setup we consider. In particular, we may encounter longer decay chains if the gluino decay to heavier neutralinos/charginos is kinematically accessible, leading to events with more and softer jets than in the simplified model. As too soft jets typically fail the cuts performed in the ATLAS and CMS analyses, this can clearly affect the constraints. However, very soft jets would mainly arise in parameter regions with a

\footnotetext{
${ }^{5}$ See [36] for a similar analysis by ATLAS.
}

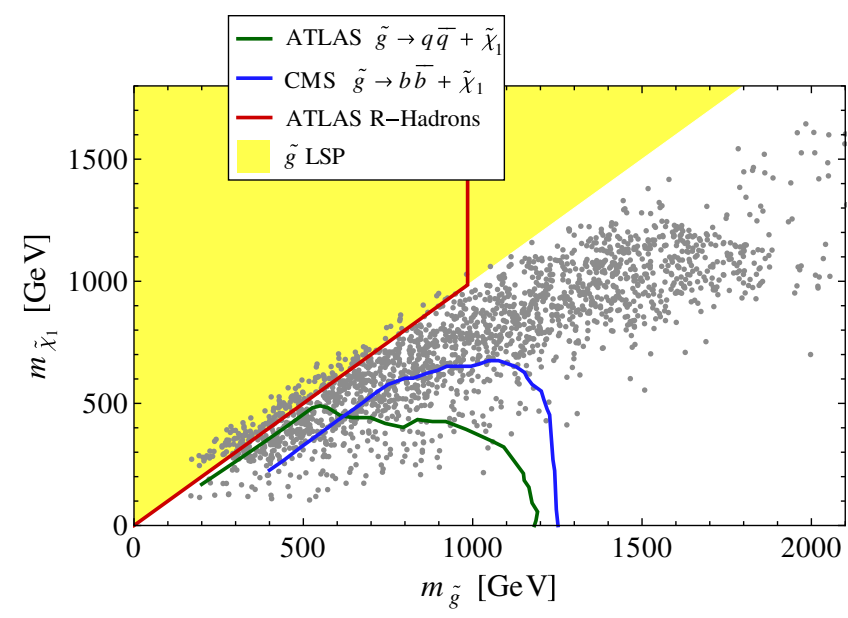

FIG. 4 (color online). Parameter points with successful gauge coupling unification (gray). Also shown are the constraints on the gluino mass for the decay modes $\tilde{g} \rightarrow q \bar{q}+\tilde{\chi}_{1}$ and $\tilde{g} \rightarrow$ $b \bar{b}+\tilde{\chi}_{1}$ by ATLAS and CMS (see text). The yellow region features a gluino LSP which is constrained by the ATLAS search for stable R-hadrons.

strongly compressed spectrum where initial state radiation is anyway required to pass the event trigger, the latter being insensitive to the gluino decay. Therefore, we believe that at least the ATLAS limit can still be used as a reasonable estimate. The stronger CMS limit should not be applied, as we do not find a preference for $b$-jets in the final state compared to light quark or gluon jets. We have, nevertheless, included it in Fig. 4 as the LHC gluon searches without $b$-tagging should reach a similar sensitivity once the full data sample collected at $\sqrt{s}=8 \mathrm{TeV}$ is analyzed. Therefore, the CMS constraint can be seen as a projection of the current sensitivity of the LHC to gluinos.

A small fraction of the benchmark points features a gluino LSP. Apart from the fact that a gluino LSP is very unfavorable from a cosmological perspective, stable gluinos with $m_{\tilde{g}}<985 \mathrm{GeV}$ are excluded by the ATLAS search for R-hadrons [37]. This constraint is also depicted in Fig. 4.

We observe that more than $90 \%$ of the benchmark points with PGU fulfill $m_{\tilde{\chi}_{1}}>0.5 m_{\tilde{g}}$. The strong compression of the spectrum implies that the limits on the gluino mass are considerably weaker than in ordinary SUSY schemes like the CMSSM. In particular we find that a large fraction of the benchmark points with $m_{\tilde{g}}=500-1000 \mathrm{GeV}$ is not excluded by the LHC. Note that it is not a general feature of mirage mediation that the collider limits on the gluino mass can be relaxed (see [24]). Only for $\varrho \lesssim 4$ the constraints become considerably weaker which is exactly the parameter regime favored by PGU.

We should also point out that the gluino can be rather long-lived in the scenario we consider. This is partly because of the phase space suppression due to the small mass difference between the gluino and neutralino, and partly because of the large mass of the squarks which 
mediate the gluino decay. The gluino decay rates in case of $m_{\tilde{\chi}_{1}} \sim m_{\tilde{g}}$ scale as (see e.g. [38])

$\Gamma\left(\tilde{g} \rightarrow q \bar{q}+\tilde{\chi}_{1}\right) \propto$ gaugino fraction $\times \frac{\left(m_{\tilde{g}}-m_{\tilde{\chi}_{1}}\right)^{5}}{m_{\tilde{q}}^{4}}$,

$$
\Gamma\left(\tilde{g} \rightarrow g+\tilde{\chi}_{1}\right) \propto \text { Higgsino fraction } \times \frac{\left(m_{\tilde{g}}-m_{\tilde{\chi}_{1}}\right)^{3} m_{t}^{2}}{m_{\tilde{q}}^{4}},
$$

where $m_{t}$ denotes the top mass and $m_{\tilde{q}}$ the mass of the squark in the intermediate state. In the setup we consider, the squarks are in the range $m_{\tilde{q}} \sim 15 \mathrm{TeV}$ as implied by the requirement $m_{h}=126 \mathrm{GeV}$ (see section III B). Note that the decay of the gluino into two quarks scales with the gaugino fraction of $\tilde{\chi}_{1}$, as the Higgsino coupling to light quarks is suppressed, while top quarks in the final state are kinematically inaccessible for $m_{\tilde{\chi}_{1}} \sim m_{\tilde{g}}$. The decay into the gluon and neutralino, on the other hand, scales with the Higgsino component of $\tilde{\chi}_{1}$. In our setup the mass splitting between left- and right-handed squarks is typically small, i.e. parity is approximately preserved in the squark sector. Therefore, the decay of the gluino into the gaugino and gluon-which requires parity violation [39] — can be neglected.

We have systematically determined the total gluino decay rate $\Gamma_{\tilde{g}}$ for the benchmark sample using the tool SDECAY 1.3 [40]; the corresponding distribution is shown in Fig. 5. ${ }^{6}$ We find that for slightly more than $10 \%$ of the benchmark points the decay length $c / \Gamma_{\tilde{g}}$ exceeds $10 \mu \mathrm{m}$ which roughly corresponds to the LHC resolution. This suggests that, in a non-negligible fraction of the parameter space, one might be able to observe displaced vertex signatures. Note that, in our approach, we assumed universal squark masses at the high scale and fixed $m_{\tilde{q}}$ such that the correct Higgs mass is obtained. There exist, however, theoretical uncertainties in the calculation of the Higgs mass at the level of a few $\mathrm{GeV}$ [41]. As the squark mass enters the loop corrections to $m_{h}$ only logarithmically this translates into an $\mathcal{O}(1)$ uncertainty on the squark mass. ${ }^{7}$ Given that $\Gamma_{\tilde{g}} \propto m_{\tilde{q}}^{-4}$, our calculation of the gluino decay rate can, thus, only be seen as an order of magnitude estimate. For many benchmark points $c / \Gamma_{\tilde{g}}$ is at least close to the LHC position resolution. Therefore, we believe that there is indeed a realistic chance to observe displaced vertices. Particularly long gluino lifetimes are obtained if the LSP is gauginolike. In this case $\Gamma_{\tilde{g}}$ is suppressed by the fifth power of the mass splitting (or the decay must proceed through the subleading Higgsino admixture of $\tilde{\chi}_{1}$ ).

\footnotetext{
${ }^{6}$ The distribution was slightly smoothed.

${ }^{7}$ Note also that in the benchmark sample we have set $\tan \beta=$ 10-50. For $\tan \beta<10$, larger squark masses are required in order to obtain $m_{h}=126 \mathrm{GeV}$; i.e. the lifetime of the gluino would be further enhanced.
}

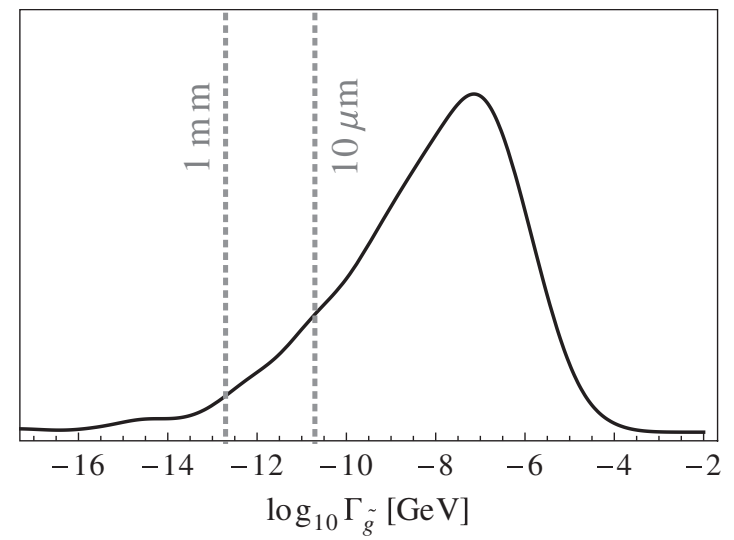

FIG. 5. Distribution of the gluino decay rate within the benchmark sample. The vertical dashed lines indicate where the gluino decay length $c / \Gamma_{\tilde{g}}$ reaches $10 \mu \mathrm{m}$ (roughly the LHC resolution) and $1 \mathrm{~mm}$ respectively.

The gluino will, however, almost certainly decay within the inner pixel detectors of ATLAS / CMS. It is unlikely that the gluino decay vertex is more than a few $\mathrm{mm}$ from the initial collision vertex (see Fig. 5). While dedicated searches for gluino decays with such tiny displacements do currently not exist, it seems feasible to employ similar methods as for the identification of $b$-jets. In particular one could study the distribution of the transverse impact parameter in events with jets and missing energy. One might worry that the jets arising from the gluino decays are too soft to be detected. However, we find that a decay length above the LHC resolution can be obtained for mass splittings $m_{\tilde{g}}-m_{\tilde{\chi}_{1}}$ as large as about $100 \mathrm{GeV}$ (depending on the composition of $\tilde{\chi}_{1}$ ). We thus want to emphasize the importance to develop search strategies for gluinos with decays slightly displaced from the collision vertex. It is also important to determine how $b$-tagging is affected if the superpartner spectrum contains gluinos with a decay length similar to that of $b$-mesons.

Even if gluinos are too short-lived to give rise to displaced vertices, the study of gluino decays is of high interest. This is because details of the superpartner sector are encrypted in the gluino decay pattern. It will be difficult to distinguish the multijet decay modes of the gluino. However, as was pointed out in [42], it is feasible to identify the radiative mode $\tilde{g} \rightarrow g+\tilde{\chi}_{1}$ which typically gives rise to events with a low jet multiplicity. This particular decay mode is very sensitive to the Higgsino component of the lightest neutralino [cf. (16)]. A large branching fraction in this mode would thus hint at a relatively small $\mu$ term. Of course, it will be challenging to determine the full set of high scale parameters just from the gluino decay. But in any case it is remarkable that gluino decays can in principle be used to learn about the spectrum of superpartners which are, in principle, outside the reach of the LHC. 


\section{B. Dark matter}

As it is well known, the lightest neutralino is a very good candidate for the dark matter in the Universe. However, in the simplest realizations of gravity mediation, the lightest neutralino is either binolike or Higgsino-like. Considerable neutralino mixing does only occur in some narrow regions of parameter space. Due to its small annihilation cross section, the bino density from thermal production typically exceeds the observed dark matter density $\Omega_{\mathrm{DM}}$ by far. The latter has recently been determined at high accuracy by the Planck Collaboration [43],

$$
\Omega_{\mathrm{DM}} h^{2}=0.1196 \pm 0.0031
$$

Higgsinos, on the other hand, undergo efficient annihilations into third generation quarks or gauge bosons; coannihilations with the charged Higgsinos further enhance their cross section. Hence, the relic density of a Higgsino LSP is typically well below the dark matter density. In both cases one has to invoke a nonstandard cosmological history if the LSP is to account for the observed dark matter. In case of the Higgsino, nonthermal production e.g. by the decay of a heavy gravitino or modulus field is a viable option (see e.g. [44-46]). For a bino LSP the situation is more unfavorable as, if its abundance is diluted by the decay of a heavy field, binos are typically regenerated by the same decay, reintroducing precisely the same problem $[47,48]$.

In mirage mediation, the gaugino masses are nonuniversal at the high scale due to the anomaly-mediated contributions. We have seen that if we require PGU, we are drawn into a region of parameter space where the gaugino spectrum is highly compressed. This turns out to be very favorable for the dark matter density. We have determined the neutralino relic density $\Omega_{\chi} h^{2}$ for a large benchmark sample using the boundaries (15) with the tool MicrOMEGAs 2.4.5 [49]. In Fig. 6 we compare the distribution of $\Omega_{\chi} h^{2}$ among the sample points with and without imposing PGU. ${ }^{8}$

We find that without the requirement of PGU, the lightest neutralino is typically bino- or Higgsino-like, similar to the models with gaugino mass unification. The distribution of the relic density among the benchmark points has two peaks corresponding to the bino and the Higgsino case respectively.

If we include the requirement of PGU, the picture changes dramatically. Remarkably, as most benchmark points with PGU feature a compressed spectrum, the distribution is now peaked close to observed dark matter density. The reason is that in the parameter regions with precision unification, the mass difference between bino and wino is typically $\sim 10 \%$, suggesting the occurrence of coannihilations. In particular in case of a bino LSP, wino pair processes would still dominate the annihilation cross section. These would be suppressed by a Boltzmann factor

\footnotetext{
${ }^{8}$ For this figure we have disregarded benchmark points with a gluino LSP. The distribution was again slightly smoothed.
}

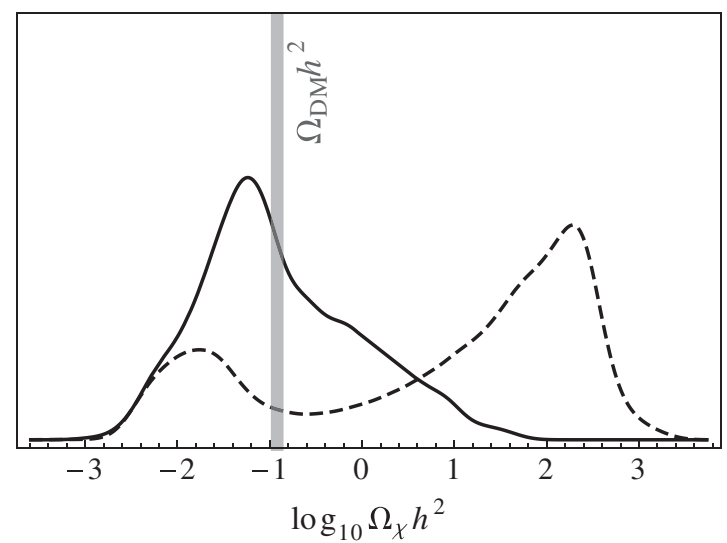

FIG. 6. Distribution of the thermal neutralino relic density for the benchmark sample with (solid) and without (dashed) requiring PGU. The observed dark matter density is indicated by the gray contour.

$$
B=\exp \left(-2 \frac{m_{\tilde{W}}-m_{\tilde{B}}}{T_{F}}\right)
$$

with the freeze-out temperature $T_{F} \sim m_{\tilde{B}} / 20$. Given a splitting of $\sim 10 \%$ one finds $B \sim 0.01$. This factor compensates for the large wino pair-annihilation cross section, yielding a relic dark matter density consistent with observation. Another possibility to obtain the correct relic density is by mixing effects in the neutralino sector.

Turning to direct dark matter detection, we find that the cross section of the lightest neutralino with nucleons is dominated by exchange of the light Higgs (as the other scalars are in the multi- $\mathrm{TeV}$ regime). The coupling of the lightest neutralino to the Higgs scales with the gaugino/ Higgsino mixing angle; it vanishes in the limit of a pure state. The neutralino proton cross section $\sigma_{p}$ for the benchmark points with PGU is shown in Fig. 7. For the determination of $\sigma_{p}$ we used MicrOMEGAs 2.4.5, but took the

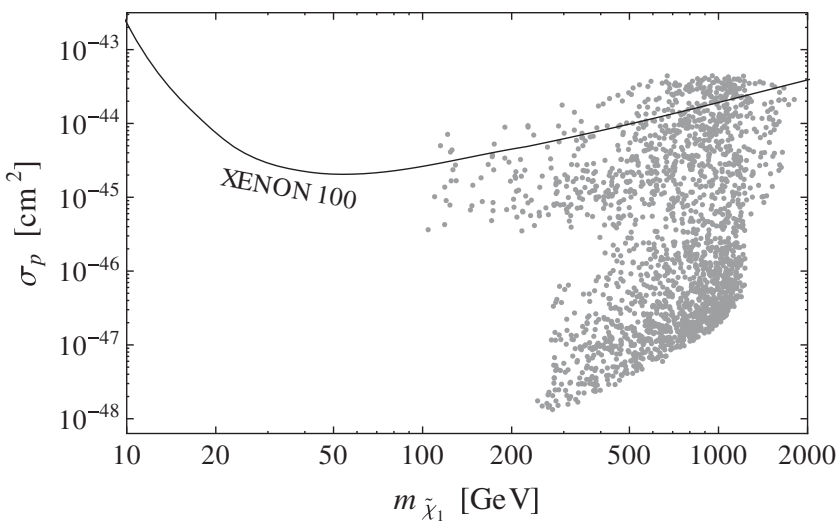

FIG. 7. Neutralino proton cross section for the benchmark points with successful gauge coupling unification (gray). The current limit from the XENON100 direct dark matter search is also shown. The latter is only applicable if the lightest neutralino accounts for all dark matter in the Universe. 
updated nuclear quark form factors from [50] suggested by recent lattice QCD calculations (see also [51]). Note that these form factors suggest a smaller strange quark contribution to the nucleon mass compared to earlier computations [52]. They thus tend to give a smaller $\sigma_{p}$.

We find that $\sigma_{p}$ scatters between $10^{-48} \mathrm{~cm}^{2}$ and $10^{-43} \mathrm{~cm}^{2}$. While the XENON100 experiment [53] has just begun to probe this regime of cross sections, the next generation of direct detection experiments will be able to test a significant fraction of the depicted benchmark points. Still, there exist benchmark points with a binolike LSP which have a strongly suppressed $\sigma_{p}$. These may even be consistent with thermal dark matter production due to coannihilations. Consequently, there is no guarantee for a signal in direct dark matter detection. One should also keep in mind that the lightest neutralino must not necessarily account for the dark matter in the Universe such that direct detection experiments would be doomed to fail.

\section{CONCLUSIONS}

This analysis is based on the hypothesis that PGU is not an accident. In general, imposing PGU reduces the dimension of the soft supersymmetry breaking parameters by 1 . Within the more "traditional" patterns of soft masses, imposing PGU implies typically a large $\mu$ parameter, which is in conflict with usual "naturalness" arguments. On the other hand, a particularly attractive pattern of soft masses, consistent with PGU, is the one of mirage mediation where the gaugino spectrum is compressed and the $\mu$ parameter can be small. Such a spectrum can be realized in various string constructions which exhibit a "competition" between gravity- and anomaly-mediated contributions to soft masses. The similarity of gaugino masses at low energies has profound implications for collider and dark matter searches.

By requiring PGU we are driven in a corner of the MSSM parameter space with a nonstandard collider phenomenology. The small mass difference between gluino and neutralino typically leads to events with a reduced

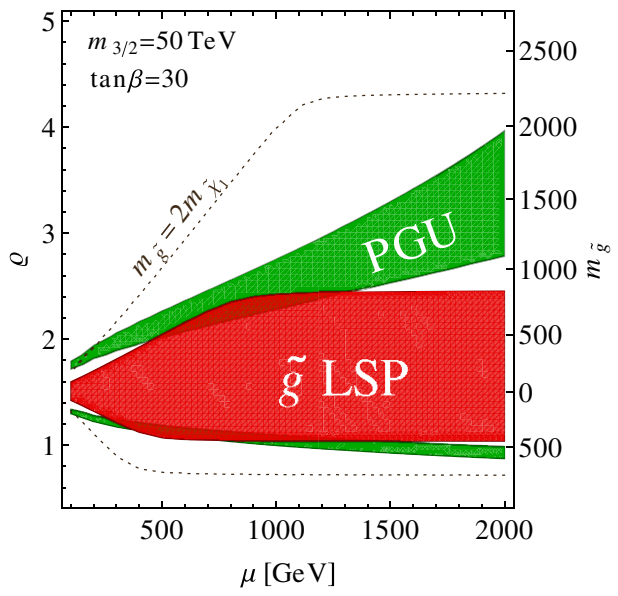

amount of visible energy. The detection of gluinos becomes far more challenging than in most of the standard SUSY models. In the case of extreme compression, initial state radiation is required to trigger on the SUSY events and the detection efficiency is strongly reduced. The gluino is typically rather long-lived; its decay length may exceed the LHC resolution and lead to displaced vertices at the level of $\mu \mathrm{m}$ to $\mathrm{mm}$. Even if displaced vertices are absent, interesting information on the superpartner spectrum can be obtained by studying the gluino decay modes.

Another virtue of compressed gaugino masses is that they generically lead to coannihilation effects in the early Universe. Cosmological problems related to the overproduction of dark matter which are present e.g. in the CMSSM can easily be solved. Indeed, we find that in the parameter space with PGU, the relic neutralino density is expected to be close to the observed dark matter density.

Finally, it is needless to say that measuring superpartner spectra that support PGU will provide us with invaluable information on how the standard model is completed in the ultraviolet.

\section{ACKNOWLEDGMENTS}

We would like to thank Jamie Tattersall and Jan Hajer for useful discussions. This work was supported by the SFB-Transregio TR33 "The Dark Universe" (Deutsche Forschungsgemeinschaft), the European Union 7th network program "Unification in the LHC era" (PITNGA-2009-237920), the Deutsche Forschungsgemeinschaft (DFG) cluster of excellence Origin and Structure of the Universe and the ERC Advanced Grant project "FLAVOUR" (267104).

\section{APPENDIX: DEPENDENCE OF PGU ON tan $\beta$ AND $m_{3 / 2}$}

Figures 8 and 9 illustrate the dependence of PGU on $\tan \beta$ and $m_{3 / 2}$.

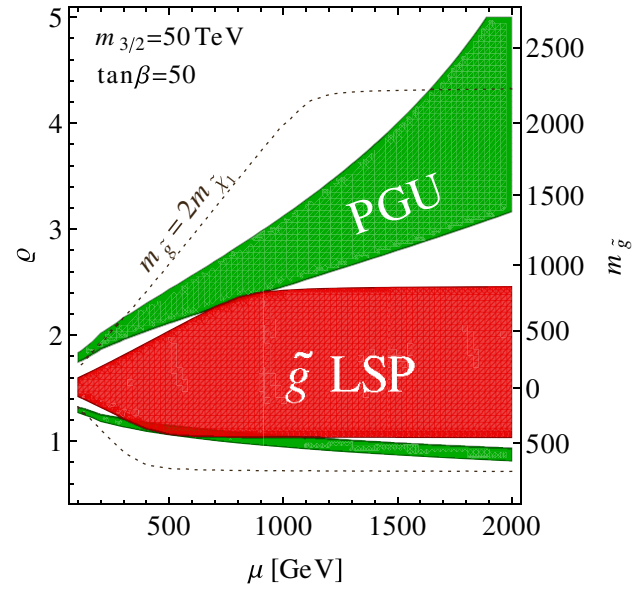

FIG. 8 (color online). Same as Fig. 3 for different choices of $\tan \beta$. 

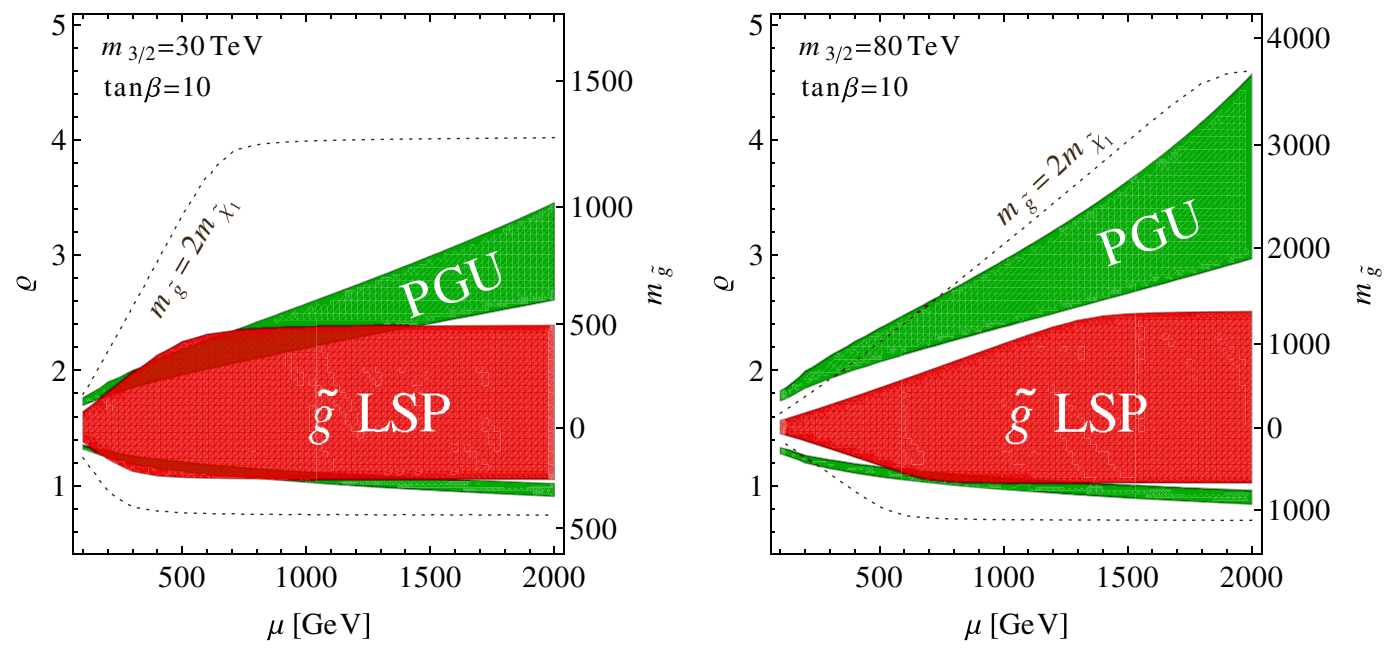

FIG. 9 (color online). Same as Fig. 3 for different choices of $m_{3 / 2}$.

[1] E. Witten, Nucl. Phys. B188, 513 (1981).

[2] S. Dimopoulos, S. Raby, and F. Wilczek, Phys. Rev. D 24, 1681 (1981).

[3] M. L. Alciati, F. Feruglio, Y. Lin, and A. Varagnolo, J. High Energy Phys. 03 (2005) 054.

[4] C. Amsler et al. (Particle Data Group), Phys. Lett. B 667, 1 (2008).

[5] M. S. Carena, L. Clavelli, D. Matalliotakis, H. P. Nilles, and C. Wagner, Phys. Lett. B 317, 346 (1993).

[6] L. Roszkowski and M. A. Shifman, Phys. Rev. D 53, 404 (1996).

[7] S. Raby, M. Ratz, and K. Schmidt-Hoberg, Phys. Lett. B 687, 342 (2010).

[8] E. Hardy, J. March-Russell, and J. Unwin, J. High Energy Phys. 10 (2012) 072.

[9] K. Choi, A. Falkowski, H. P. Nilles, and M. Olechowski, Nucl. Phys. B718, 113 (2005).

[10] K. Choi, K. S. Jeong, and K.-i. Okumura, J. High Energy Phys. 09 (2005) 039.

[11] A. Falkowski, O. Lebedev, and Y. Mambrini, J. High Energy Phys. 11 (2005) 034.

[12] O. Lebedev, H. P. Nilles, and M. Ratz, Phys. Lett. B 636, 126 (2006)

[13] V. Löwen and H.P. Nilles, Phys. Rev. D 77, 106007 (2008).

[14] M. S. Carena, S. Pokorski, and C. Wagner, Nucl. Phys. B406, 59 (1993).

[15] A. Lahanas and K. Tamvakis, Phys. Lett. B 348, 451 (1995).

[16] A. Anandakrishnan and S. Raby, arXiv:1303.5125.

[17] B. Allanach, Comput. Phys. Commun. 143, 305 (2002).

[18] S. Bethke, Nucl. Phys. B, Proc. Suppl. 234, 229 (2013).

[19] N. Arkani-Hamed and S. Dimopoulos, J. High Energy Phys. 06 (2005) 073.

[20] S. Kachru, R. Kallosh, A. D. Linde, and S. P. Trivedi, Phys. Rev. D 68, 046005 (2003).
[21] K. Choi, A. Falkowski, H. P. Nilles, M. Olechowski, and S. Pokorski, J. High Energy Phys. 11 (2004) 076.

[22] E. Dudas, A. Linde, Y. Mambrini, A. Mustafayev, and K. A. Olive, Eur. Phys. J. C 73, 2268 (2013).

[23] S. Krippendorf, H. P. Nilles, M. Ratz, and M. W. Winkler, Phys. Lett. B 712, 87 (2012).

[24] M. Badziak, S. Krippendorf, H. P. Nilles, and M.W. Winkler, J. High Energy Phys. 03 (2013) 094.

[25] B. L. Kaufman, B. D. Nelson, and M. K. Gaillard, Phys. Rev. D 88, 025003 (2013).

[26] B. S. Acharya, G. Kane, and P. Kumar, Int. J. Mod. Phys. A 27, 1230012 (2012).

[27] H. Abe, T. Higaki, T. Kobayashi, and Y. Omura, Phys. Rev. D 75, 025019 (2007).

[28] R. Kallosh and A. D. Linde, J. High Energy Phys. 02 (2007) 002.

[29] J. L. Feng, K. T. Matchev, and T. Moroi, Phys. Rev. Lett. 84, 2322 (2000).

[30] J. L. Feng, K. T. Matchev, and T. Moroi, Phys. Rev. D 61, 075005 (2000).

[31] T. J. LeCompte and S. P. Martin, Phys. Rev. D 84, 015004 (2011).

[32] T. J. LeCompte and S. P. Martin, Phys. Rev. D 85, 035023 (2012).

[33] H. K. Dreiner, M. Kramer, and J. Tattersall, Europhys. Lett. 99, 61001 (2012).

[34] ATLAS Collaboration, Report No. ATLAS-CONF-2012109, 2012.

[35] S. Chatrchyan et al. (CMS Collaboration), arXiv:1305.2390.

[36] ATLAS Collaboration, Report No. ATLAS-CONF-2012145.

[37] G. Aad et al. (ATLAS Collaboration), Phys. Lett. B 720, 277 (2013).

[38] R. Barbieri, G. Gamberini, G. F. Giudice, and G. Ridolfi, Nucl. Phys. B301, 15 (1988). 
[39] H.E. Haber and G.L. Kane, Nucl. Phys. B232, 333 (1984).

[40] M. Mühlleitner, A. Djouadi, and Y. Mambrini, Comput. Phys. Commun. 168, 46 (2005).

[41] P. Draper, J. Feng, P. Kant, S. Profumo, and D. Sanford, Phys. Rev. D 88, 015025 (2013).

[42] R. Sato, S. Shirai, and K. Tobioka, J. High Energy Phys. 11 (2012) 041.

[43] P. Ade et al. (Planck Collaboration), arXiv:1303.5076.

[44] T. Moroi and L. Randall, Nucl. Phys. B570, 455 (2000).

[45] M. Fujii and K. Hamaguchi, Phys. Lett. B 525, 143 (2002).

[46] R. Kitano, H. Murayama, and M. Ratz, Phys. Lett. B 669, 145 (2008)
[47] T. Moroi, M. Yamaguchi, and T. Yanagida, Phys. Lett. B 342, 105 (1995).

[48] S. Nakamura and M. Yamaguchi, Phys. Lett. B 638, 389 (2006).

[49] G. Belanger, F. Boudjema, A. Pukhov, and A. Semenov, Comput. Phys. Commun. 176, 367 (2007).

[50] G. Belanger, F. Boudjema, A. Pukhov, and A. Semenov, arXiv:1305.0237.

[51] A. Thomas, P. Shanahan, and R. Young, Nuovo Cimento Soc. Ital. Fis. 035C, 3 (2012).

[52] M. Pavan, I. Strakovsky, R. Workman, and R. Arndt, PiN Newslett. 16, 110 (2002).

[53] E. Aprile et al. (XENON100 Collaboration), Phys. Rev. Lett. 109, 181301 (2012). 